\title{
Users' requirements and preferences in the success of a new product: a case study of automotive design
}

\begin{abstract}
There is a significant amount of research and empirical investigations indicating that consideration of usersô requirements and preferences has become an important issue in product design. They are also important as a valuable source of facilitating the product designer in establishing the characteristics of a new product. The aim of this study is to establish the influence of usersôrequirements and preferences that has resulted in the success of an automotive. A survey was conducted to identify the key characteristics factors for success of an automotive. Based on these findings, several recommendations are proposed for a product designer to develop the specifications for a new automotive.
\end{abstract}

Keyword: Product success; Requirements and preferences; User 